\title{
Descripción Histológica de los Diferentes Segmentos del Apa- rato Digestivo de Avestruz (Struthio camelus var. domesticus)
}

\author{
Histologic Description of the Different Segments from the Ostrich Digestive System \\ (Struthio camelus var. domesticus) \\ ${ }^{* * * *}$ Illanes, J.; ${ }^{* *}$ Fertilio, B; **Chamblas, M.; "Leyton, V. \& *Verdugo, F.
}

ILLANES, J.; FERTILIO, B.; CHAMBLAS, M.; LEYTON, V. \& VERDUGO, F. Descripción histológica de los diferentes segmentos del aparato digestivo de avestruz (Struthio camelus var. domesticus). Int. J. Morphol., 24(2):205-214, 2006.

RESUMEN:La expansión y rápido desarrollo de la crianza de ratites, ha implicado desafíos científicos y técnicos para enfrentar este nuevo tipo de explotación en Chile. El avestruz (Struthio camelus var. domesticus), es un ave herbívora, forrajera y altamente selectiva, que presentan semejanzas y diferencias con otras aves y herbívoros, entre ellos los rumiantes. Se conoce bastante sobre su anatomía gastrointestinal, sin embargo, su histología no ha sido investigada en profundidad. Para este análisis, se obtuvieron muestras de los diferentes segmentos del tubo digestivo de 6 avestruces adultas, clínicamente sanas. Se procesaron de acuerdo a las técnicas de histología corriente y analizadas al microscopio óptico. El avestruz muestra un esófago con pliegues longitudinales en toda su extensión y en su mucosa glándulas tubuloalveolares simples y ramificadas. Un proventrículo (estómago glandular) con glándulas tubulares simples y ramificadas en la mucosa y tubuloalveolares compuestas en la submucosa; un refuerzo de tejido conjuntivo compacto de haces paralelos, entre la serosa y la capa muscular longitudinal externa. Un intestino delgado, con abundantes vellosidades y ausencia de células de Paneth. Un ciego que, en su cara luminal, presenta un pliegue en espiral. El análisis histológico comparativo permitió establecer que la estructura básica de la pared del tubo digestivo es semejante a la descrita en otras especies.

PALABRAS CLAVE: Avestruz; Histología; Aparato Digestivo.

\section{INTRODUCCIÓN}

Struthio camelus domesticus (Swart, 1987) es descrita como reflejo del híbrido natural de avestruces de granja en Sudáfrica, la que actualmente se conoce como avestruz de cuello negro o African Black. Esta ave fue desarrollada mediante programas de mejoramiento genético, con el objetivo de aumentar el valor comercial de la especie. Se caracteriza por ser de menor talla y más fértil que las otras subespecies, estructura del plumaje bien desarrollada, carácter dócil, y de fácil crianza en granjas, ya que es tremendamente curiosa y amigable con los humanos (Deeming, 2001; Camiruaga, 2004). En relación a las características anatómicas generales del tracto digestivo, Camiruaga \& Simonetti, (2003) señalan que el avestruz presenta semejanzas y diferencias, tanto con otras aves, como con los rumiantes y otros herbívoros (equinos). Del análisis comparativo con la gallina, presenta ciertas diferencias anatómicas, una de ellas es no presentar buche, órgano almacenador de alimento que existe en otras aves. El proventrículo y el estómago muscular (molleja), en el avestruz, pueden cumplir dicha función (Angel, 1996). No presentan vesícula biliar, por lo que el vaciamiento de la bilis se realiza directamente al intestino delgado. Además, el intestino grueso del avestruz, a diferencia de otras especies, representa el $50 \%$ del largo total del tubo digestivo y el intestino delgado corresponde sólo al 35,5\%. (Camiruaga, 2004). En el presente trabajo se analizó la histología normal de los diferentes segmentos del tubo digestivo del avestruz: esófago, proventrículo, estómago muscular (molleja o ventrículo), intestino delgado (duodeno, yeyuno e íleon) e intestino grueso (ciego, colon y recto), y se analizó comparativamente con especies a las que se le asocia morfológicamente, como las aves domésticas, rumiantes, seudo rumiantes (camélidos) y algunos otros herbívoros.

\footnotetext{
" Facultad de Medicina. Clínica Alemana-Universidad del Desarrollo, Chile.

"* Facultad de Medicina Veterinaria. Universidad Iberoamericana de Ciencia y Tecnología, Chile.
} 


\section{MATERIAL Y MÉTODO}

El material biológico empleado en este trabajo, corresponde a muestras de los diferentes segmentos del tubo digestivo, obtenidas desde 6 avestruces adultas clínicamente sanas, con un rango de 12 a 14 meses de edad, provenientes de criaderos de la VI Región de Chile. El material proviene del frigorífico FAENAGRO ubicado en Graneros, ruta 5 sur, km. 74, VI Región. Obtenidas las muestras de los diferentes segmentos del aparato digestivo del avestruz: esófago, proventrículo, estómago muscular, intestino delgado (duodeno, yeyuno e íleon) e intestino grueso (ciego, colon y recto), fueron fijadas en formalina tamponada al $10 \%$, seguido del procedimiento habitual de técnica histológica. Los cortes se realizaron de $5 \mu$ de grosor, fueron observados, fotografiados y analizados bajo microscopio de luz (Olympus BX41 con cámara digital Olympus C-5060 Wide zoom, 5.1 mega pixel). Con las fotografías, se realizó un análisis comparativo de los aspectos histológicos encontrados en avestruz con aquellos descritos en otras especies. La tinción empleada fue la técnica corriente de hematoxilina-eosina (HE) y de Van Gieson, con la finalidad de observar los tejidos muscular y conjuntivo (Luna, 1968).

\section{RESULTADOS}

Descripción general del tubo digestivo del avestruz: Los segmentos que conforman el tubo digestivo corresponden al esófago, proventrículo (estómago glandular), estómago muscular (ventrículo o molleja), intestino delgado, ciego e intestino grueso. La pared del tubo digestivo presenta cuatro capas o túnicas concéntricas: mucosa; submucosa, muscular y serosa y/o adventicia. A lo largo del tubo digestivo, existen diferencias histológicas de cada capa, principalmente en la túnica mucosa, las cuales se manifiestan por las características funcionales de cada segmento. La mucosa presenta un epitelio superficial, una lámina propia o corion y una muscular de la mucosa. Además, en el epitelio superficial se abren innumerables glándulas ubicadas principalmente en la lámina propia, la cual corresponde a un estrato de tejido conjuntivo laxo, muy vascularizado y rico en nódulos linfáticos. Entre la lámina propia y la submucosa encontramos la muscular de la mucosa, constituida por dos o tres capas de tejido muscular liso, dispuestas de modo circular y longitudinal, no siempre bien definida. La submucosa está compuesta por tejido conjuntivo laxo o denso, donde se pueden observar glándulas, vasos sanguíneos y plexos nerviosos submucosos. La muscular está compuesta por dos capas de células musculares lisas, una interna circular y otra externa longitudinal, conteniendo, además, una red de fibras nerviosas (plexos nerviosos mientéricos) y vasos sanguíneos. La serosa es la capa más externa del tubo digestivo. Está compuesta por tejido conjuntivo laxo cubierto por un epitelio plano, que co- rresponde al mesotelio. Contiene, además, algunos vasos sanguíneos, fibras nerviosas y tejido adiposo.

1. Esófago: El esófago es la primera porción del tubo digestivo, el cual anatómicamente se ubica entre la orofaringe y el estómago glandular o proventrículo. Tiene una longitud promedio de $90 \mathrm{~cm}$ en un avestruz adulta. Al corte transversal muestra un lumen irregular, en su pared se observan varios pliegues de disposición longitudinal a modo de columnas, los que van aumentando de grosor hacia la porción distal (Fig. 1). Estos pliegues están formados por un eje central de mucosa y submucosa (Figs. 1a y 1b). La mucosa está constituida por un epitelio plano estratificado no queratinizado, que muestra un estrato basal germinativo, uno intermedio poliédrico y uno superficial plano. Bajo el epitelio continúa una gruesa capa de tejido conjuntivo denso, el cual corresponde al corion o lámina propia, la que presenta abundantes glándulas de secreción mucosa de tipo tubuloalveolar simple y ramificada, en toda su extensión, con un conducto excretor que atraviesa el epitelio y desemboca en el lúmen. Se observa además, un gran número de nódulos linfáticos. La muscular de la mucosa presenta sólo una capa gruesa de fibras musculares lisas longitudinales y determina el límite entre el corion y la submucosa (Figs. 1b, 1c y 1d). La submucosa es poco desarrollada, constituida de tejido conjuntivo laxo, presenta plexos nerviosos submucosos a lo largo de su estructura y numerosos vasos sanguíneos (Figs. 1a y 1b). La muscular está conformada por una gruesa capa circular interna de músculo liso y otra longitudinal externa más irregular y delgada. Entre ambas capas musculares se observa una delgada banda de tejido conjuntivo laxo, muy irrigada y con plexos nerviosos mientéricos (Fig. 1a). La serosa no presenta ninguna diferenciación con respecto a lo habitual.

2. Proventrículo (estómago glandular): Macroscópicamente el proventrículo presenta una forma de medialuna, de color rosado pálido, con una ligera cubierta de grasa. El proventrículo del avestruz es un órgano que presenta un lumen amplio y en su pared se pueden reconocer 4 capas: mucosa, submucosa, muscular y serosa (Figs 2 y 2a). La mucosa presenta numerosas vellosidades, cuya superficie se observa cubierta por una gran cantidad de mucus (glicocáliz). El epitelio de revestimiento de secreción mucosa es cilíndrico simple en toda la superficie. En las criptas vacían su secreción las glándulas fúndicas tubulares simples y ramificadas, con células principales y parietales. Entre ellas, se encuentra un abundante corion de tejido conjuntivo laxo. Luego se observa la muscular de la mucosa de tejido muscular liso, que une este estrato con la submucosa. En la submucosa se encuentran glándulas de tipo tubuloalveolar compuesta, con células parietales y que desembocan en las criptas, donde liberan la secreción a la luz del proventrículo, mediante conductos excretores de epitelio cilíndrico simple. En la submucosa del proventrículo se observan fibras nerviosas que corresponden a plexos submucosos (Fig. 2a). La muscular en su mayor parte está representada por la capa circular interna de 


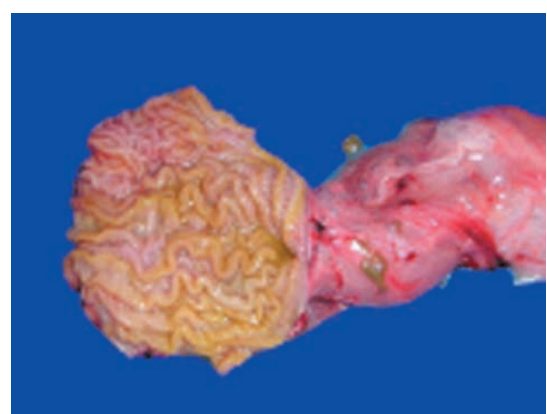

Fig. 1. Aspecto macroscópico de esófago. Hacia el lumen muestra los pliegues longitudinales y externamente, la adventicia.

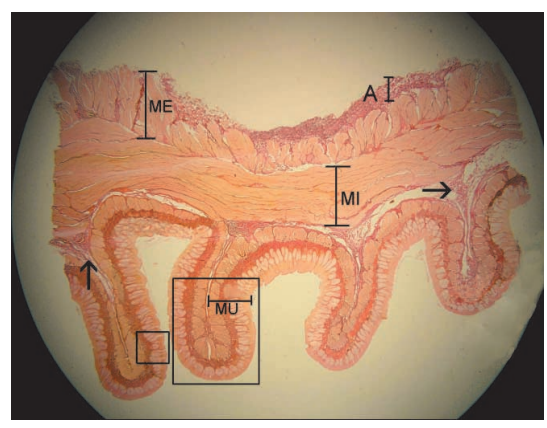

Fig. 1a. Corte panorámico transversal de esófago. MU. Mucosa; Submucosa (flecha); MI, muscular interna; ME. Muscular externa; A. Adventicia. Nótese los pliegues longitudinales. Los recuadros mayor y menor se muestran a mayor aumento en las Fig. 1b y 1c, respectivamente. Van Gieson 12.5X.

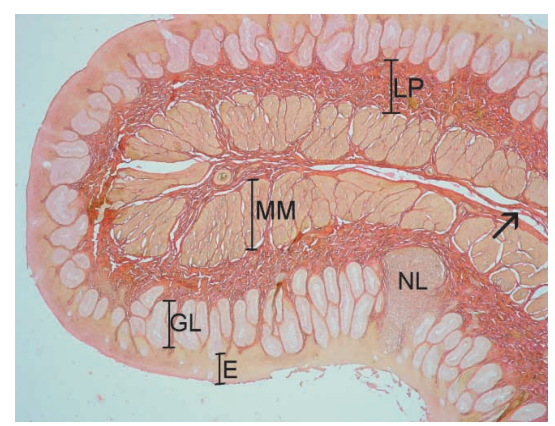

Fig. 1b. Corte histológico de un pliegue de esófago. E. Epitelio plano estratificado no queratinizado; GL. Glándulas tubuloalveolares simples; LP. Lámina propia; MM. Muscular de la mucosa; NL. Nódulo linfático; Submucosa (flecha). Van Gieson 100X.

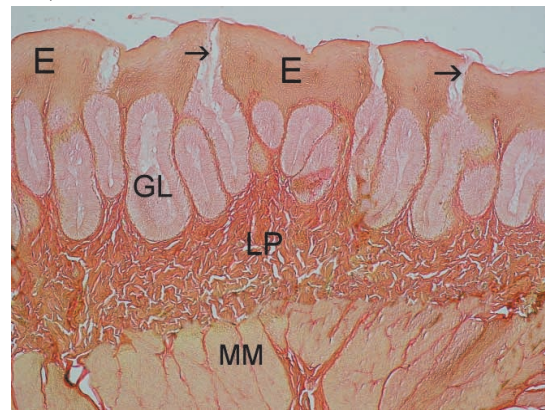

Fig. 1c. Corte histológico de la mucosa de esófago. E. Epitelio plano estratificado no queratinizado; GL. Glándulas tubuloalveolares simples; Conductos excretores (flechas); LP. Lámina propia; MM. Muscular de la mucosa. Van Gieson 200X.

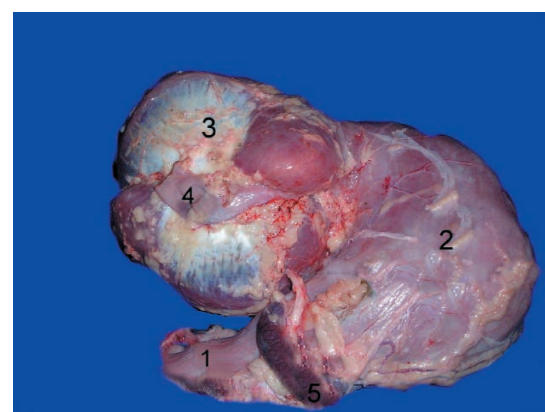

Fig. 2. Aspecto macroscópico de proventrículo y su relación con otros órganos. 1. Porción final del esófago; 2 Proventrículo; 3 . Estómago muscular; 4. Porción inicial del duodeno; 5. Bazo.

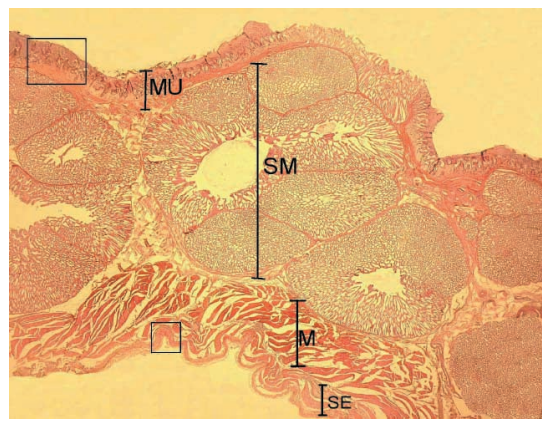

Fig. 2a. Corte histológico panorámico de la pared de proventrículo. MU. Mucosa; SM. Submucosa; M. Muscular; SE. Serosa. Los recuadros mayor y menor se muestran a mayor aumento en las Figs. $2 \mathrm{~b}$ y $2 \mathrm{c}$, respectivamente. H-E 12.5X.

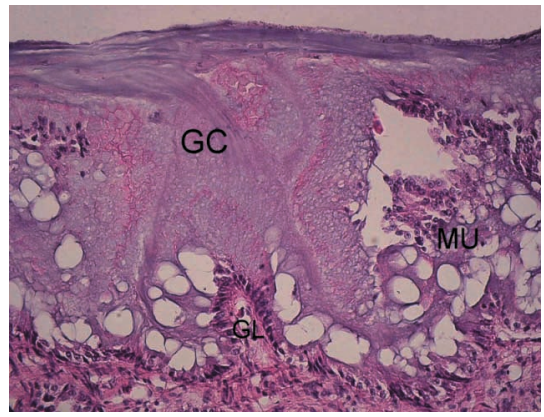

Fig. 2b. Corte histológico de la mucosa de proventrículo. GC. Glicocáliz; GL. Glándulas fúndicas; MU. Mucosa. H-E 200X.

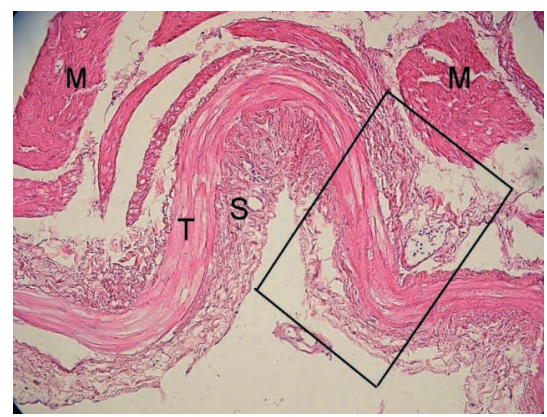

Fig. 2c. Corte histológico de proventrículo. M. Muscular; T. Capa tendínea; S. Serosa. El recuadro se muestra a mayor aumento en la Fig. 2d. H-E 200X.

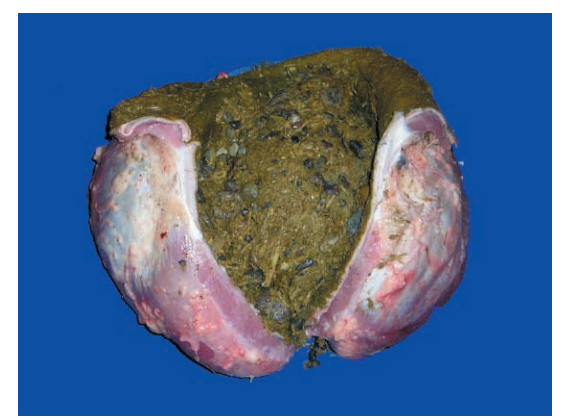

Fig. 3. Aspecto macroscópico de estómago muscular abierto del avestruz.

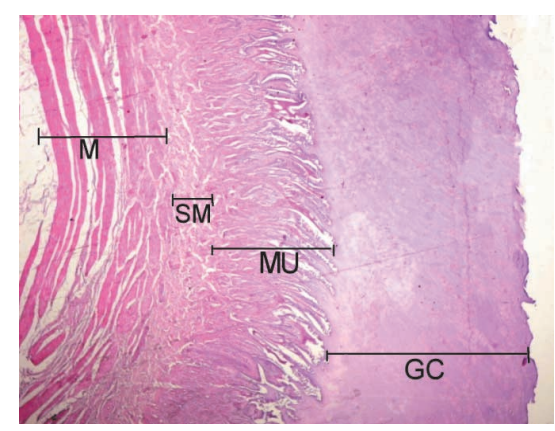

Fig. 3a. Corte histológico panorámico de la pared de estómago muscular. GC. Glicocáliz; MU Mucosa; SM Submucosa; M. Muscular. H-E $12.5 \mathrm{X}$

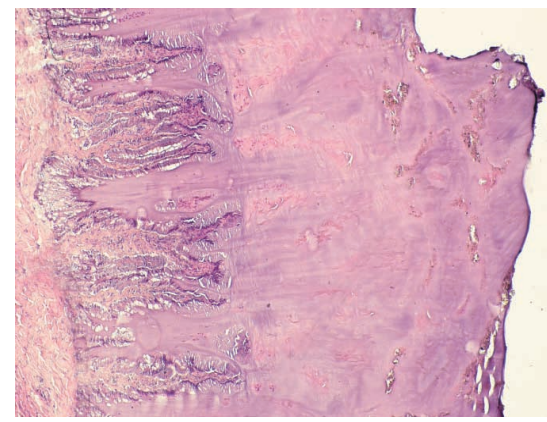

Fig. 3b. Corte histológico de estómago muscular. Muestra la mucosa cubierta por el glicocáliz secretado por las glándulas tubuloalveolares simples y ramificadas. H-E 100X.

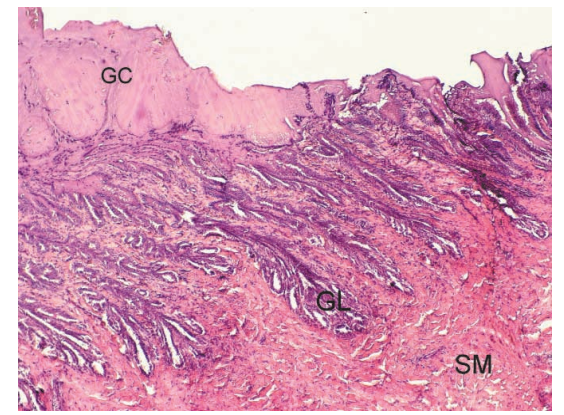

Fig. 3c. Corte histológico de estómago muscular Muestra en la mucosa: GC. Glicocáliz; GL. Glándulas tubuloalveolares simples y ramificadas; SM. Submucosa. H-E 200X. 
músculo liso, unida a la submucosa. Luego una capa más delgada, no bien definida, la longitudinal externa. Entre ambas capas encontramos células ganglionares correspondientes a los plexos mientéricos. La serosa constituida por el mesotelio y tejido conjuntivo laxo que recubren la pared del proventrículo. Entre las capas muscular y serosa, encontramos una gruesa lámina de tejido conjuntivo compacto de haces paralelos, esta capa tendínea rodea todo el órgano (Figs 2a, 2c y 2d).

3. Estómago muscular (molleja): Macroscópicamente, el estómago muscular se observa como un órgano compacto pequeño, en comparación al largo total del tubo digestivo, presenta una longitud promedio de $40 \mathrm{~cm}$ (Fig. 3). La mucosa está tapizada por un grueso estrato de aspecto córneo, el cual corresponde a la cutícula (glicocáliz). Presenta gran cantidad de arrugas en su superficie. Bajo ella se observa un epitelio cilíndrico simple, el que se invagina en la lámina propia, formando numerosas glándulas tubuloalveolares simples y ramificadas de secreción mucosa, cuyos conductos se abren en la cutícula. La muscular de la mucosa se observa como una delgada capa de fibras musculares lisas, de desarrollo variable, visible en algunos sectores y ausente en otros. Estas fibras musculares corren paralelas al eje longitudinal y, en algunas porciones, se confunde con la túnica muscular. La submucosa está formada por tejido conjuntivo denso irregular. Se observan tabiques que se ramifican hacia la capa muscular (Figs. 3a, 3b y 3c). La muscular es gruesa, muestra tres capas de musculatura lisa que corresponden a una capa plexiformes interna, una capa transversal media y una gruesa capa longitudinal externa. Entre las diferentes capas se observan bandas de tejido conjuntivo denso y grandes vasos sanguíneos. Entre las capas musculares se observan numerosos plexos mientéricos (Figura 3a y 3d). La serosa no presenta ninguna diferenciación con respecto a lo habitual.

4. Intestino delgado: El intestino delgado del avestruz adulta mide, en promedio, 7,5 m de longitud. Consta de tres segmentos denominados duodeno, yeyuno e íleon. No existen límites definidos entre ellos (Fig. 4). Su estructura básica es similar a lo largo de toda su extensión, pudiendo encontrarse características diferentes de acuerdo a su función.

4.1. Duodeno: Macroscópicamente, corresponde a un asa de color gris rojizo plegada en U, situada entre el estómago muscular y el yeyuno. Se observan proyecciones de mucosa y submucosa correspondientes a válvulas conniventes (Figs. 4.1a y 4.1b). La mucosa ocupa gran parte de la pared intestinal. Está constituida por numerosas vellosidades recubiertas por un epitelio cilíndrico simple con chapa estriada, es decir microvellosidades ubicadas en el borde apical de la célula. En la base de las vellosidades se observan numerosas criptas intestinales. El corion de tejido conjuntivo laxo muestra nódulos linfáticos y vasos sanguíneos. El epitelio de revestimiento de las vellosidades y de las criptas, muestra células caliciformes.
La muscular de la mucosa es delgada y está constituida por una capa circular interna y otra longitudinal externa. La capa circular interna emite ramas hacia la lámina propia hasta el vértice de las vellosidades (Figs. 4.1c y 4.1d). La submucosa, poco desarrollada corresponde a tejido conjuntivo laxo, observándose en ella vasos sanguíneos y plexos nerviosos submucosos (Fig. 4.1b). La muscular muestra una capa circular interna y una longitudinal externa notablemente más delgada. Entre ambas capas se observan numerosos plexos nerviosos mientéricos y vasos sanguíneos (Fig. 4.1c). La serosa no presenta ninguna diferenciación con respecto a lo habitual.

4.2. Yeyuno: El análisis macroscópico de este segmento, reveló la ausencia de válvulas conniventes descritas en el duodeno. Sin embargo, la estructura histológica básica de este segmento es similar a la del duodeno (Figs. 4.2a, 4.2b y 4.2c). La mucosa muestra largas vellosidades, con fibras musculares lisas provenientes de la muscular de la mucosa. Con un epitelio de revestimiento cilíndrico simple con chapa estriada, se aprecia un aumento de las células caliciformes presentes en el epitelio y una disminución de las criptas intestinales. En la lámina propia se observan nódulos linfáticos y vasos sanguíneos. La muscular de la mucosa es delgada y corresponde a dos capas de fibras musculares lisas, una interna de disposición circular y otra externa longitudinal (Figs. 4.2a, 4.2b, 4.2c, 4.2d y 4.2e). La submucosa es poco desarrollada, presenta vasos sanguíneos y plexos nerviosos submucosos. La muscular es más gruesa que la observada en el segmento anterior, con una capa circular interna y una capa longitudinal externa más delgada (Fig. 4.2b). La serosa no presenta ninguna diferenciación con respecto a lo habitual.

4.3 Ileon: La mucosa del íleon muestra menor cantidad de vellosidades, más cortas y más gruesas que en los segmentos anteriores. Con un epitelio cilíndrico simple, con chapa estriada y células caliciformes. Se observan numerosas criptas intestinales. Las vellosidades intestinales están formadas por un eje central de tejido conjuntivo laxo y fibras musculares lisas provenientes de ramas de la muscular de la mucosa. En la lámina propia, se observan vasos sanguíneos y nódulos linfáticos. La muscular de la mucosa presenta tres capas de musculatura lisa, una circular interna, una intermedia longitudinal y otra circular externa (Figs. 4.3a, 4.3b, 4.3c y 4.3d). La submucosa es poco desarrollada, presenta vasos sanguíneos y plexos nerviosos submucosos. La muscular se dispone en una capa circular interna delgada y otra longitudinal externa más gruesa, entre ambas se aprecian plexos mientéricos (Fig. 4.3e). La serosa no presenta ninguna diferenciación con respecto a lo habitual.

5. Intestino grueso: El intestino grueso del avestruz adulto mide aproximadamente $16 \mathrm{~m}$ de longitud y representa la última porción del tubo digestivo. Presenta tres segmentos: ciego, colon y recto. La estructura histológica general es similar en toda su longitud, mostrando diferencias en sus segmentos de acuerdo a su función. 


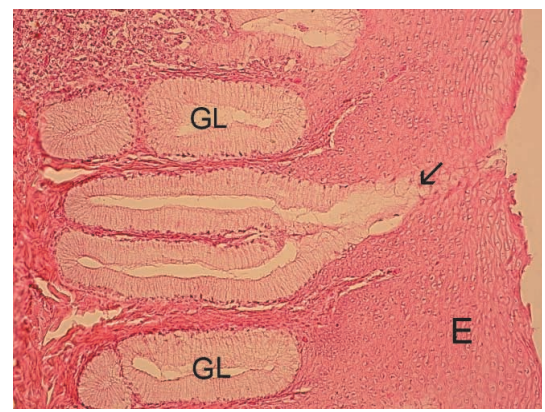

Fig. 1d. Corte histológico de la mucosa esofágica. E. Epitelio plano estratificado no queratinizado; GL. Glándulas tubuloalveolares simples y ramificadas; Conducto excretor (flecha). H-E, 400X.

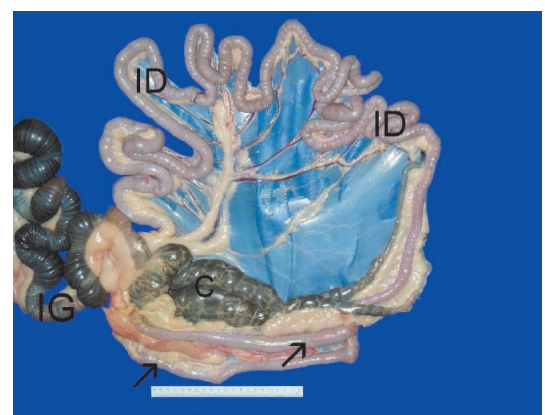

Fig. 4. Aspecto macroscópico de intestino. ID Intestino delgado; IG. Intestino grueso ; Duodeno (flechas); C. Ciegos.

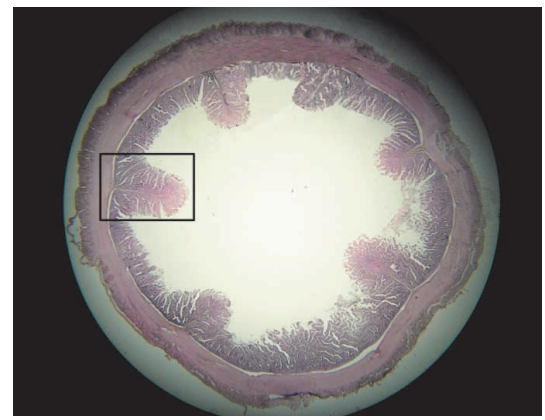

Fig. 4.1a. Corte panorámico transversal de duodeno. El recuadro se muestra a mayor aumento en la Fig. 4.1b. H-E 12.5X.

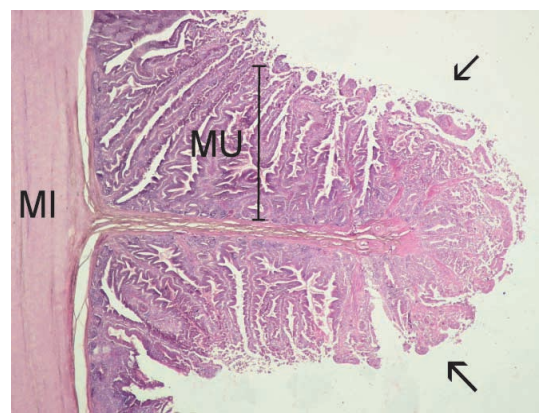

Fig. 4.1b. Corte histológico de una válvula connivente de duodeno. MU. Mucosa; MI. Muscular interna; Válvula connivente (flechas). H-E $100 X$.

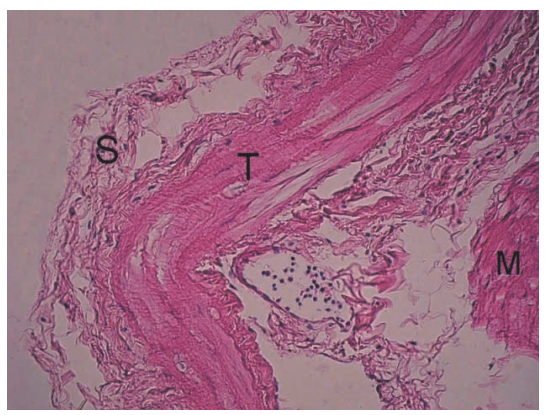

Fig. 2d. Corte histológico de proventrículo. M. Muscular; T. Capa tendínea; S. Serosa. H-E 400X.

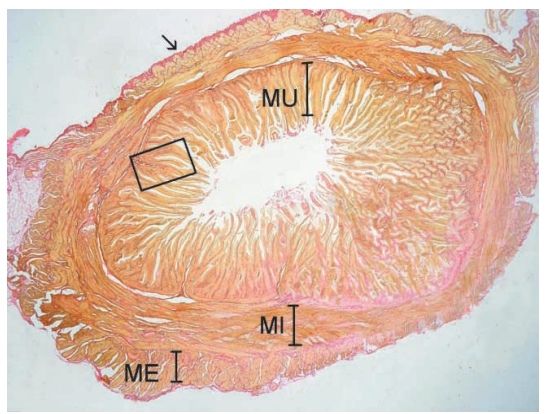

Fig. 4.2a. Corte panorámico transversal de yeyuno. MU. Mucosa; MI. Muscular interna; ME. Muscular externa; Serosa (flechas). El recuadro se muestra a mayor aumento en la Fig. 4.2d. Van Gieson 12.5X.

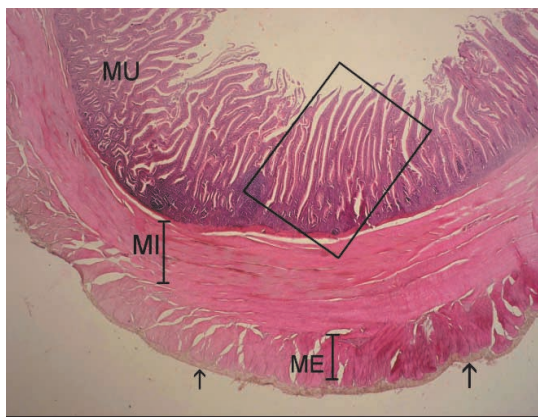

Fig. 4.2b. Corte histológico de yeyuno. MU. Mucosa; MI. Muscular interna; ME Muscular externa; Serosa (flechas). El recuadro se muestra a mayor aumento en la Fig. 4.2c. H-E 40X.

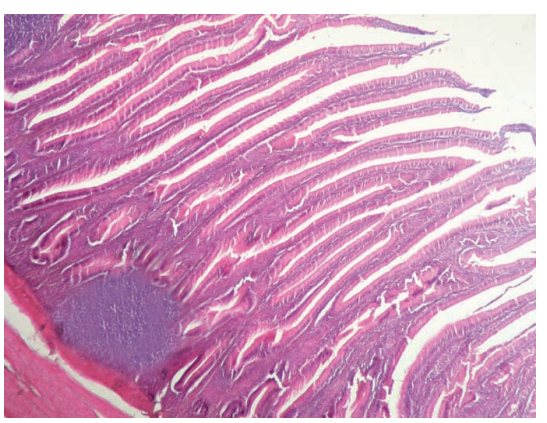

Fig. 4.2c. Corte histológico de yeyuno. Muestra la mucosa con sus vellosidades, criptas intestinales poco profundas, nódulo linfoide y muscular de la mucosa. H-E 100X.

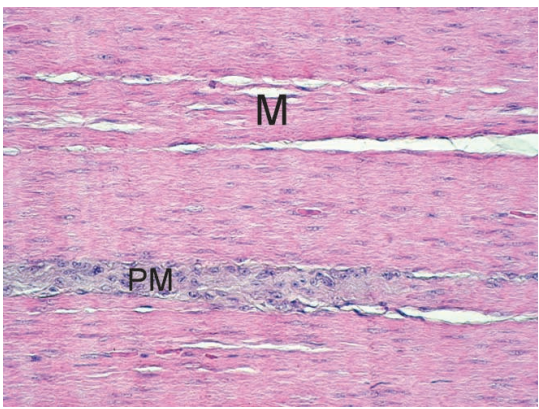

Fig. 3d. Corte histológico de estómago muscular. M. Muscular; PM. Plexo nervioso mientérico. H-E 400X

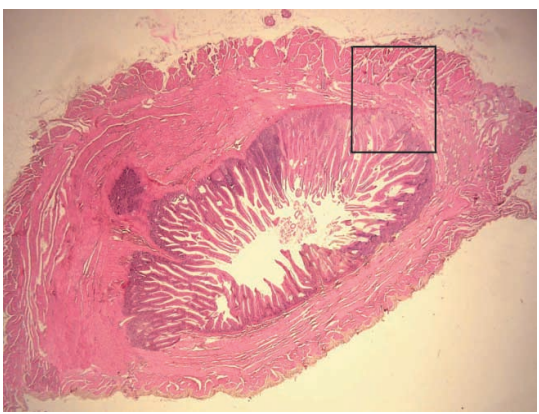

Fig. 4.3a. Corte panorámico transversal de ileon. El recuadro se muestra a mayor aumento en la Fig. 4.3b. H-E 12.5X

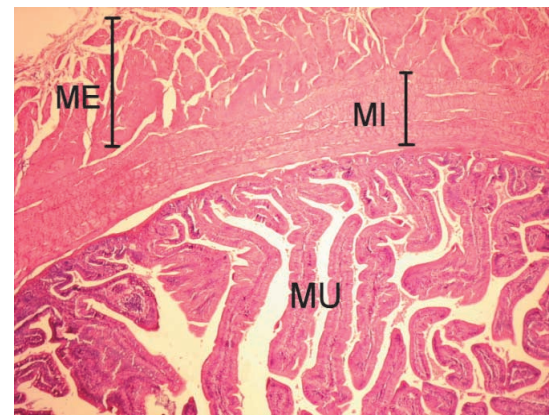

Fig. 4.3b. Corte histológico de ileon. MU. Mucosa; MI. Muscular interna; ME. Muscular externa. H-E $100 \mathrm{X}$.

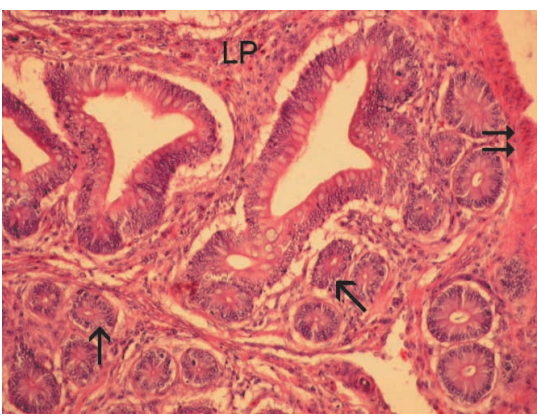

Fig. 4.3c. Corte histológico de la mucosa de ileon. LP. Lamina propia; Criptas intestinales (flechas) y muscular de la mucosa (doble flecha). H-E 200X. 
5.1. Ciego: Corresponde a dos grandes dilataciones, que miden, en promedio, $90 \mathrm{~cm}$ de longitud cada una, ellas marcan la transición entre el intestino delgado y el intestino grueso. Macroscópicamente, la porción proximal del ciego, muestra hacia el lumen un largo pliegue en espiral, con un núcleo de mucosa y submucosa (Figs. 4 y 5.1a). La porción distal, muestra pliegues longitudinales formados por mucosa y submucosa (Figs. 5.1b). La mucosa presenta una superficie luminal lisa carente de vellosidades intestinales con un epitelio cilíndrico simple. Sin embargo, muestra numerosas criptas intestinales y numerosas células caliciformes, más abundantes que en el intestino delgado. Se observan nódulos linfáticos aislados. La muscular de la mucosa corresponde a dos capas de fibras musculares lisas, una circular interna y otra longitudinal externa (Figs. 5.1b, 5.1c y 5.1d). La submucosa está formada por una delgada capa de tejido conjuntivo laxo. Presenta, además, vasos sanguíneos que aumentan su espesor, plexos nerviosos submucosos y gran cantidad de tejido adiposo (Figs. 5.1 y y 5.1c). La muscular presenta menor desarrollo que en el intestino delgado, está formada por una delgada capa circular interna y longitudinal externa. En ellas, se observan plexos nerviosos mientéricos. Entre ambas capas musculares se observa tejido adiposo y vasos sanguíneos (Fig. 5.1b). La serosa no presenta ninguna diferenciación con respecto a lo habitual.

5.2. Colon: Macroscópicamente, presenta pliegues o válvulas conniventes hacia el lumen, los cuales están formados por núcleos de mucosa y submucosa (Fig. 4). La mucosa del colon es de menor grosor que la del intestino delgado y carece de vellosidades intestinales. Con un epitelio cilíndrico simple y menor número de criptas intestinales, que las observadas en el ciego, con numerosas células caliciformes. Se observan algunos nódulos linfáticos aislados. La muscular de la mucosa es delgada y muestra una capa circular interna y otra longitudinal externa. La capa circular interna presenta ramificaciones hacia la lámina propia (Figs. 5.2a, 5.2b, 5.2c y 5.2d). La submucosa está constituida de tejido conjuntivo laxo. Presenta vasos sanguíneos, tejido adiposo y plexos nerviosos submucosos (Figs. 5.2b y 5.2c). La muscular está constituida por dos capas de musculatura lisa, una interna de disposición circular y una externa más delgada de disposición longitudinal. Entre ellas se disponen plexos nerviosos mientéricos (Fig. 5.2b). La capa serosa de tejido conjuntivo laxo, presenta la particularidad de formar saculaciones, las cuales siguen la dirección de los sacos que se forman en la capa muscular.

5.3. Recto: Se observan hacia el lumen largos pliegues longitudinales a modo de columnas, que presentan un núcleo de mucosa y submucosa (Figuras 4, 5.3a y 5.3b). La mucosa es lisa, carente de vellosidades, con un epitelio cilíndrico simple. Las criptas intestinales son más profundas que en el intestino delgado, con un mayor número de células caliciformes que en los segmentos anteriores. La muscular de la mucosa es delgada y está formada por una capa de fibras musculares lisas de disposición circular (Figura 5.3b, 5.3c y 5.3d). La submucosa está formada por tejido conjuntivo laxo. Su estructura es similar a la de los demás segmentos (5.3b y 5.3c). La muscular es gruesa, y está formada por musculatura lisa, una interna de disposición circular, y otra externa de disposición longitudinal (5.3a y 5.3b). La serosa no presenta ninguna diferenciación con respecto a lo habitual.

\section{DISCUSIÓN}

Esófago: El epitelio de la mucosa esofágica en el avestruz es de tipo plano estratificado no queratinizado, mientras que en los rumiantes la mucosa presenta un alto grado de paraqueratinización, la que es leve en el caballo (Stinson \& Calhoun, 1993). La lámina propia en el avestruz, muestra numerosas glándulas túbuloalveolares simples y ramificadas de secreción mucosa, dispuestas a lo largo de toda su extensión. La gallina, presenta glándulas de secreción mucosa de tipo túbuloacinar compuestas (Dieter Dellmann \& Eurell, 1998). Por el contrario, Delhon et al. (1984), señalan que la forma de estas glándulas es muy variable entre las diversas porciones del esófago, pasando de glándulas que tienden a formar acinos en la porción cervical del buche, a tubulares en la región torácica. En otras especies, como el rumiante, caballo y cerdo, la mucosa carece de estas glándulas (Bacha \& Wood, 1991). La muscular de la mucosa en el avestruz se observa como una gruesa capa de fibras musculares lisas de disposición longitudinal. La gallina presenta dos capas, una interna de disposición circular, formada por fascículos delgados que no siempre son apreciables, y otra externa longitudinal muy desarrollada (Delhon et al.). Según Dieter Dellmann \& Eurell, la muscular de la mucosa de las aves domésticas está formada sólo por una capa de fibras musculares lisas dispuestas longitudinalmente. En el avestruz, la submucosa esofágica, al igual que en la gallina, carece de glándulas (Dieter Dellmann \& Eurell), mientras que en el caballo y en rumiantes, estas glándulas están presentes, pero sólo se observan en la unión faringoesofágica (Stinson \& Calhoun; Bacha \& Wood). La muscular del esófago del avestruz, al igual que en la gallina, posee dos capas de musculatura lisa de disposición circular interna y longitudinal externa (Delhon et al.; (Dieter Dellmann \& Eurell). En el rumiante está constituida por músculo estriado, el que se extiende desde el esófago hasta el surco reticular; y en el caso del caballo este músculo estriado comprende los dos tercios proximales del esófago, pero va cambiando gradualmente a músculo liso en el tercio distal (Stinson \& Calhoun). El avestruz macho utiliza el esófago para ocasionar un sonido retumbante, cuando lo infla con aire que posteriormente deja escapar (Asociación Gemial de Criadores de Avestruces de Chile, 2005). 


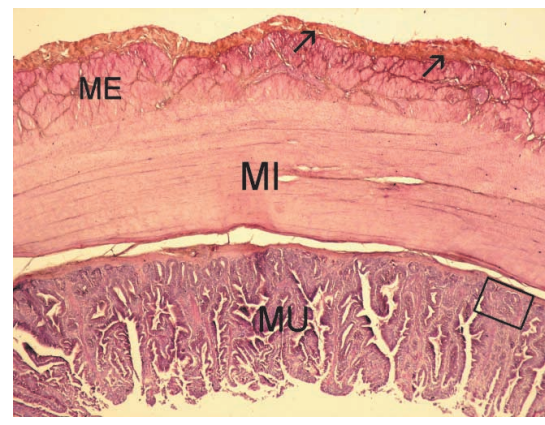

Fig. 4.1c. Corte histológico de la pared de duodeno. MU. Mucosa; MI. Muscular interna; ME. Muscular externa; Serosa (flechas). El recuadro se muestra a mayor aumento en la Fig. 4.1d. H-E $100 \mathrm{X}$.

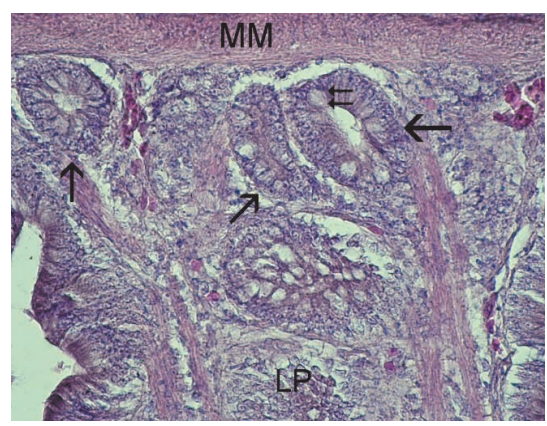

Fig. 4.1d. Corte histológico de duodeno. LP. Lámina propia; MM. Muscular de la mucosa; Criptas intestinales (flechas) y células caliciformes (doble flechas). H-E 400X

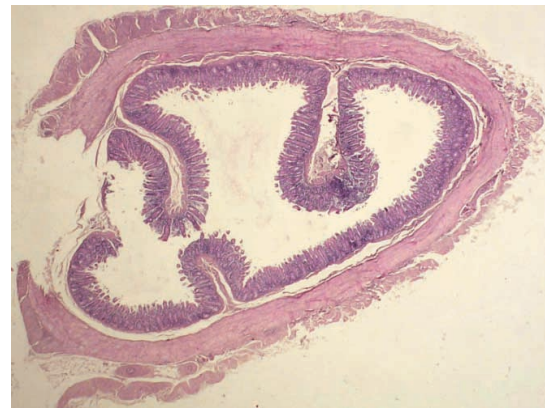

Fig. 5.1a. Corte panorámico transversal de la porción distal de ciego. H-E $12.5 \mathrm{X}$.

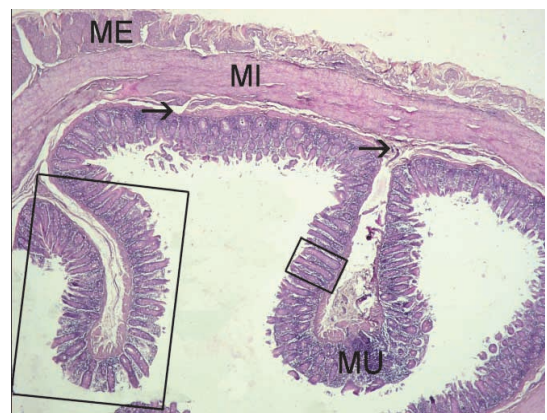

Fig. 5.1b. Corte histológico de la porción distal de ciego. MU. Mucosa; MI. Muscular interna; ME. Muscular externa y submucosa (flechas). Los recuadros mayor y menor se observan en las figuras 5.1c y 5.1d respectivamente. H-E 100X.

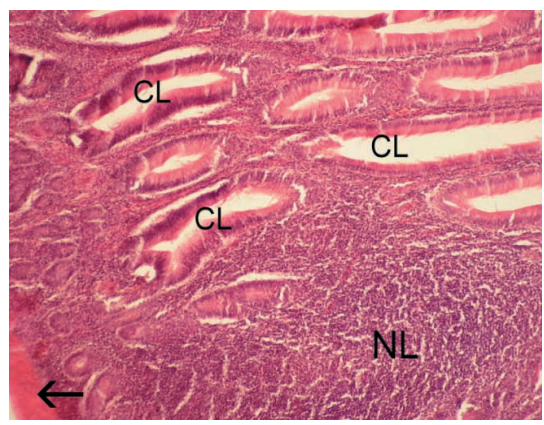

Fig. 4.2d. Corte Histológico de yeyuno. CL. Cripta intestinal ; Nódulo linfático; Muscular de la mucosa (flecha). H-E 200X.

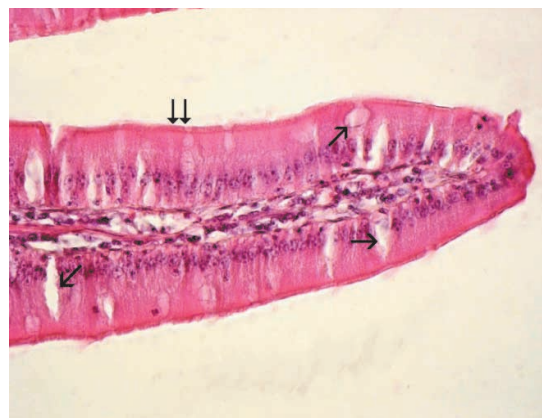

Fig. 4.2e. Corte histológico de una vellosidad de yeyuno. Células caliciformes (flechas) y chapa estriada (doble flecha). H-E 400X.

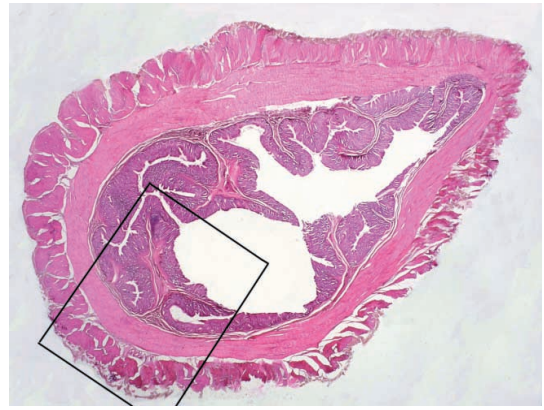

Fig. 5.2a. Corte Histológico panorámico de colon. El recuadro se muestra a mayor aumento en la Fig. 8a. H-E 12.5X.

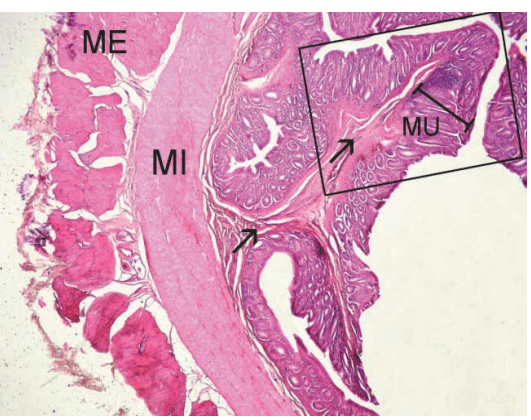

Fig. 5.2b. Corte histológico de colon. MU. Mucosa; Submucosa (flecha); MI. Muscular interna; ME. Muscular externa. El recuadro se muestra a mayor aumento en la Fig. 5.2c. H-E 100X.

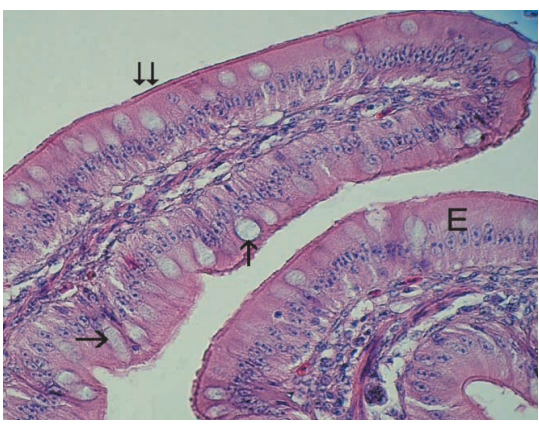

Fig. 4.3d. Corte histológico de una vellosidad de ileon. E. Epitelio; Células caliciformes (flechas) y chapa estriada (doble flecha). H-E 400X.

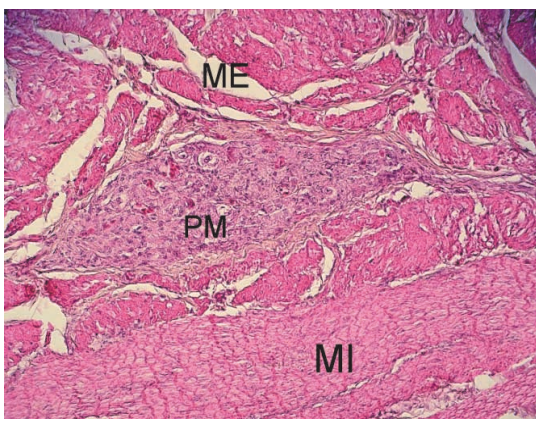

Fig. 4.3e. Corte histológico de la túnica muscular de ileon. MI. Muscular interna; PM. Plexo mientérico; ME. Muscular externa. H-E 400X.

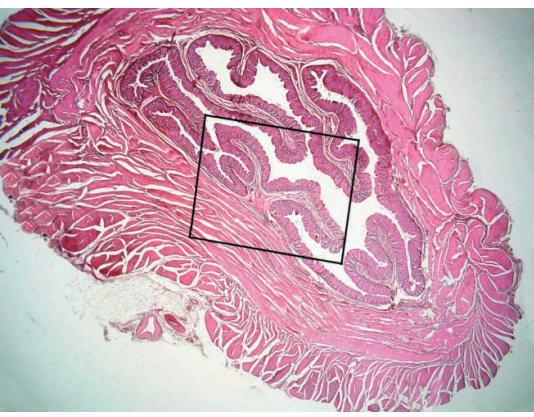

Fig. 5.3a. Corte Histológico panorámico de recto. El recuadro se muestra a mayor aumento en la Fig. 5.3b. H-E 12.5X

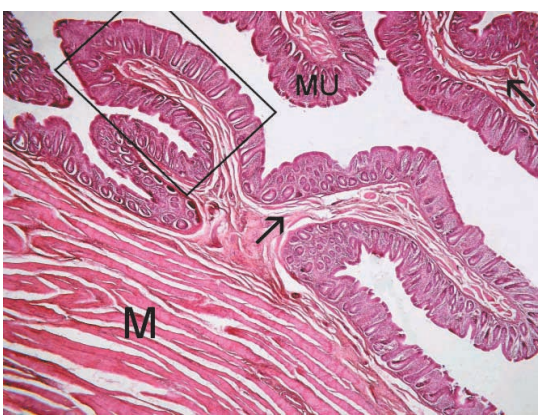

Fig. 5.3b. Corte histológico de recto. MU. Mucosa; M. Muscular; Submucosa (flechas). El recuadro se muestra a mayor aumento en la Fig. 5.3c. H-E 100X. 
Proventrículo (estómago glandular): El proventrículo en el avestruz, es el primer estómago, posee una enorme capacidad de distensión y es donde se inicia la digestión de las proteínas mediante la secreción de pepsinógeno y ácido clorhídrico, dado en parte por la secreción de glándulas fúndicas tubulares simples y ramificadas poco desarrolladas de la mucosa. El mayor aporte de ácido clorhídrico, aparentemente, es producto de las glándulas túbuloalveolares compuestas de la submucosa. En la mucosa de la llama, se observan profundas glándulas fúndicas con abundantes células parietales secretoras de ácido clorhídrico (Illanes et al., 1994). Entre la capa muscular y la serosa, encontramos una gruesa lámina de tejido conjuntivo compacto de haces paralelos; esta capa tendínea rodea todo el órgano. Tal estructura fue observada en un camélido suramericano adulto, la llama (Lama glama) por Illanes et al.

Estómago muscular (molleja): En el avestruz, la molleja y el proventrículo se observan como órganos pequeños, en relación al largo total del tubo digestivo. Desde el punto de vista histológico, la descripción de las diferentes capas de la molleja en la gallina, pato y ganso no difieren (McLelland, 2000). El estómago muscular se localiza detrás del esternón y del hígado, en el lumen contiene arena, piedras y otros materiales que ayudan a moler los alimentos más duros y gruesos. La válvula pilórica es bien desarrollada y posee un esfínter muscular muy sensible, y que restringe el tamaño de las partículas, asegurando que las partículas más grandes sean molidas, fácilmente digeridas y absorbidas en el intestino. (Camiruaga \& Simonetti; Cooper \& Mahroze, 2004). En el avestruz, la mucosa, se encuentra tapizada por un grueso estrato de aspecto córneo, que corresponde a la cutícula (glicocáliz), semejante a lo descrito en el gallo adulto (Delhon et al.). McLelland, se refiere a la cutícula, como a la membrana del estómago muscular, la cual se describe como un recubrimiento queratinoide o koilin. Microscópicamente, esta membrana en el avestruz está formada por columnas verticales de secreción, procedentes de glándulas tubulares. Bacha \& Wood confirman que éstas glándulas secretan un material córneo, generalmente denominada sustancia queratinoide; parecida a la queratina, pero que químicamente no es equivalente a ésta. En el avestruz, el epitelio de revestimiento corresponde a un epitelio cilíndrico simple, el que se invagina en la lámina propia formando numerosas glándulas túbuloalveolares simples y ramificadas, de secreción mucosa, cuyos conductos se abren en la cutícula. En el caso de la gallina, estas glándulas son de tipo tubulares ramificadas (Bacha \& Wood). En aves no domésticas, como por ejemplo el cardenal rojo, se describe la mucosa de la molleja con el mismo tipo de epitelio, con pliegues de altura variable, cubierto por la cutícula, y en la lámina propia, parecido a lo que se observa en el avestruz, se describen glándulas tubulares simples (Catroxo et al., 1997). En la base de la mucosa, en el avestruz se observa la muscular de la mucosa, la cual es de desarrollo variable lo que hace que sea visible en algunas porciones, hallazgo que coincide con lo descrito en esta especie por Bezuidenhout (1993), citado por Cooper \& Mahroze (2004) y en la lechuza (De Oliveira \& Inforzato, 1998). En cambio en otras aves domésticas y no domésticas la literatura no la describe (Catroxo et al.; Dieter Dellmann \& Eurell). La muscular, en el avestruz es semejante a la gallina, presenta tres capas gruesas de fibras musculares lisas. Sin embargo, en la gallina, en algunos cortes de molleja sólo se puede apreciar una capa de fibras musculares lisas de disposición circular (Delhon et al.).

Intestino delgado: Desde el punto de vista histológico, el intestino delgado del avestruz, comparado con el de la gallina y de otras especies, muestra ciertas similitudes y diferencias en sus segmentos. Es así como en el avestruz, en toda la mucosa del intestino delgado, observamos largas vellosidades intestinales descritas también en gallina. En los rumiantes, son estructuras cortas y gruesas (Bacha \& Wood). En los rumiantes y en el cerdo, se observan en el fondo de las criptas intestinales, células granulares eosinofílicas llamadas células de Paneth (Stinson \& Calhoun). Según Bezuidenhout, citado por Cooper \& Mahroze, estas células no fueron observadas en las avestruces. La muscular de la mucosa en el duodeno y yeyuno del avestruz, presenta dos capas de fibras musculares lisas, una circular interna y otra longitudinal externa, y en el íleon se describen tres capas de fibras musculares; en cambio en la gallina la muscular de la mucosa consiste en sólo una capa de fibras musculares lisas de disposición longitudinal a lo largo de todo el segmento (Delhon et al.). En el avestruz, la submucosa, al igual que en la gallina, se observa como una delgada capa de tejido conjuntivo laxo, muestra un mayor espesor cuando contiene plexos nerviosos submucosos o vasos sanguíneos y no muestra glándulas (Dieter Dellmann \& Eurell y McLelland). En cambio en los rumiantes y en el caballo la submucosa muestra glándulas tubuloalveolares, cuyos conductos excretores atraviesan la muscular de la mucosa y se abren en el fondo de las criptas intestinales. Estas glándulas son de tipo mucoso en los rumiantes y de tipo seroso en el caballo, las que se observan hasta el yeyuno (Stinson \& Calhoun ). La muscular consta de dos capas de musculatura lisa, una de disposición circular interna y otra longitudinal externa, en todas las especies descritas (Stinson \& Calhoun). El avestruz no escapa a esta característica.

Intestino grueso: El intestino grueso, es el último segmento del tubo digestivo y su papel primordial es la absorción de agua (Crossley, 2001). Presenta dos ciegos bien desarrollados, con una gran cantidad de especies de bacterias anaeróbicas fermentadoras, similar al contenido del estómago de los rumiantes, que les permite utilizar una buena proporción de la fibra contenida en su dieta y aprovechar la energía de los forrajes (Camiruaga \& Simonetti). En el ciego del avestruz se describe un pliegue en espiral, formado por un núcleo de mucosa y submucosa, éste juega un papel importante en la absorción de ácidos grasos volátiles y otros metabolitos producidos por la fermentación microbiana de la celulosa y hemicelulosa, Bezuidenhout, citado por Cooper \& 


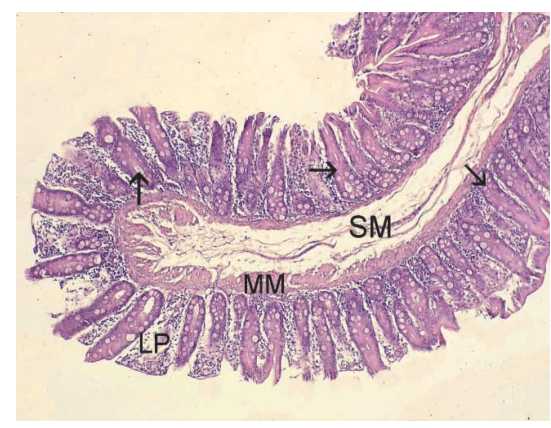

Fig. 5.1c. Corte histológico de ciego. LP. Lámina propia; MM. Muscular de la mucosa; SM. Submucosa; Criptas intestinales (flechas). H-E 200X.

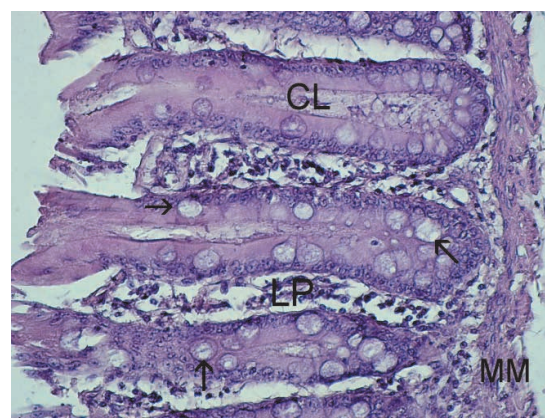

Fig. 5.1d. Corte histológico de la mucosa de ciego. LP. Lámina propia; MM. Muscular de la mucosa; CL. Criptas intestinales; Células caliciformes (flechas). H-E 400X.

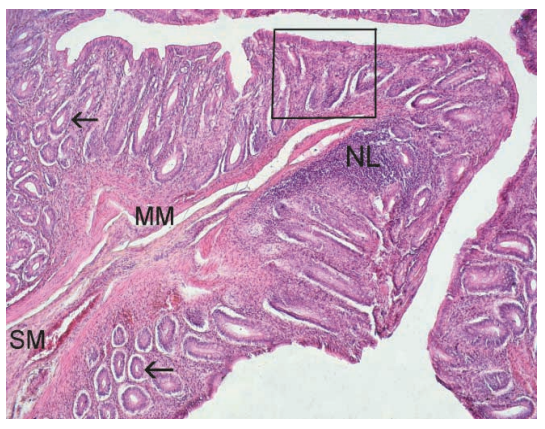

Fig. 5.2c. Corte histológico de colon. H-E 200X MM. Muscular de la mucosa; SM. Submucosa; NL. Nódulo linfático; Criptas intestinales (flechas). El recuadro se muestra a mayor aumento en la Fig. 5.2d.

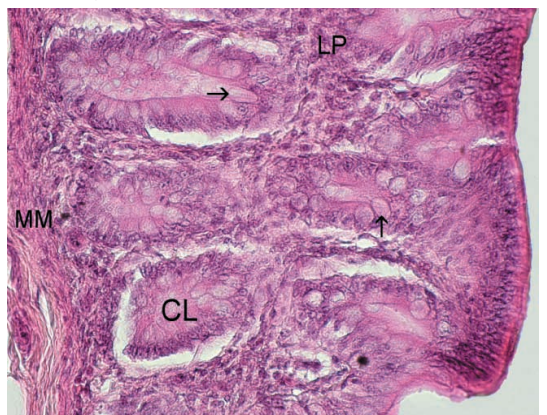

Fig. 5.2d. Corte histológico de la mucosa de colon. LP. Lámina propia; CL Cripta intestinal; MM. Muscular de la mucosa; Células caliciformes (flechas). H-E 400X

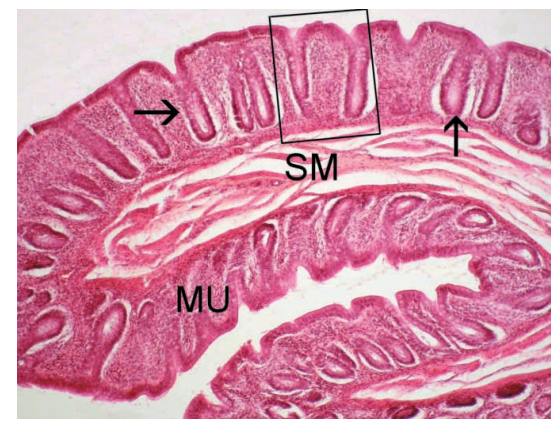

Fig. 5.3c. Corte histológico de recto. MU. Mucosa; SM. Submucosa; Criptas intestinales (flechas). El recuadro se muestra a mayor aumento en la Fig. 5.3d. H-E 200X.

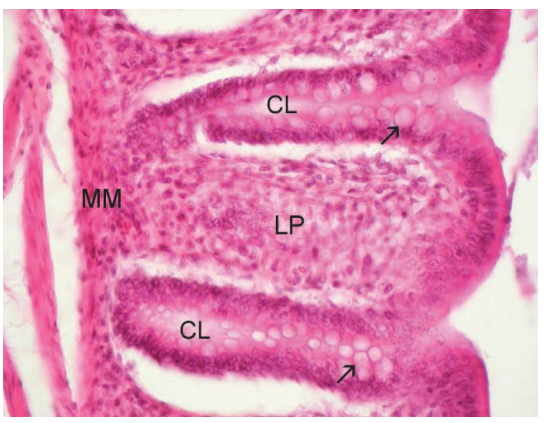

Fig. 5.3d. Corte histológico de recto. CL. Cripta intestinal; LP. Lámina propia; MM. Muscular de la mucosa; células caliciformes (flechas). H-E $400 \mathrm{X}$.
Mahroze. El colon y el recto presentan pliegues longitudinales constituidos por núcleos de mucosa y submucosa, al igual que lo descrito para otras especies domésticas (Stinson \& Calhoun). El colon del avestruz es largo, pudiendo alcanzar hasta $16 \mathrm{~m}$ en los ejemplares adultos (Cooper \& Mahroze). La mucosa en el intestino grueso del avestruz, carece de vellosidades y está formada por un epitelio de revestimiento cilíndrico simple, similar a lo descrito en el caballo y en el rumiante (Stinson \& Calhoun). En la gallina, a semejanza con las especies mencionadas anteriormente, el epitelio de revestimiento del intestino grueso, en general corresponde a un epitelio cilíndrico simple, pero la presencia de vellosidades es motivo de controversia. Calhout (1954), citado por McLelland que describió la presencia de vellosidades bien desarrolladas en la parte proximal del ciego, en la parte media más cortas y anchas, y en la parte distal cortas o ausentes. Esto fue confirmado mas tarde por (Stinson \& Calhoun). Kappelhoff (1959), citado por McLelland, comunica que las vellosidades están restringidas solo a la parte proximal del ciego. En el colon y recto se describen vellosidades intestinales mas cortas y gruesas en la gallina (Bacha, 1998). En el avestruz, al igual que en el caballo, rumiante y cerdo, la lámina propia presenta escaso tejido conjuntivo laxo a lo largo de todo el intestino grueso, debido a la presencia de gran cantidad de criptas intestinales, que son muy profundas. Existe, además, un incremento en el número de células caliciformes (Stinson \& Calhoun). En la gallina se describen criptas intestinales menos profundas, las cuales no sólo se abren entre las vellosidades, sino también en las áreas planas que hay entre ellas (Delhon et al.). En el cerdo y en el caballo, la capa longitudinal externa de la muscular del ciego y colon forma las tenias, que corresponden a grandes bandas musculares aplanadas que contienen numerosas fibras elásticas (Stinson \& Calhoun; Sisson, 2000), en el avestruz, rumiante y gallina estas estructuras no están presente (Stinson \& Calhoun; McLelland; Habel, 2000). De acuerdo al análisis microscópico realizado en los diferentes segmentos del tubo digestivo del avestruz, muestra la estructura general presente en la pared del tubo digestivo de los vertebrados. Sin embargo, se observan ciertas diferencias que pueden ser producto de cambios funcionales y de adaptación. Este análisis histológico es un aporte no solo a la biología, sino también puede ser de gran utilidad, especialmente para comprender mejor los procesos de carácter alimentario y nutritivo durante la crianza y el manejo del avestruz.

AGRADECIMIENTOS: A Frigorífico Faenagro, por su cooperación, tanto con el material biológico como su disposición, sin ello no hubiera sido posible este estudio. 
ILlANES, J.; FERTILIO, B.; CHAMbLAS, M.; LEYTON, V. \& VERDUGO, F. Histologic description of the different segments from the ostrich digestive system (Struthio camelus domesticus). Int. J. Morphol., 24(2):205-214, 2006.

SUMMARY: The rapid expansion and development of the ratites upbringing, it has implied scientific challenges and technicians to face this new type of farms systems in Chile. The ostrich (Struthio camelus var. domesticus), is an herbivore bird, forages eater and highly selective that present likeness and differences, with other birds and herbivores, among them the ruminant ones. Enough envelopes their gastrointestinal anatomy is known, however its histology has not been investigated in depth. For this analysis it was obtained samples of the different segments of the tubular digestive tract of 6 clinically healthy ostriches. They were processed according to the techniques of average histology and analyzed to the optic microscope. The ostrich show an esophagus with longitudinal pleats in all their extension and in the mucosa there are simple and branching tubulosaccular glands. The proventriculus (glandular stomach) present a simple and branching tubular glands in the mucosa and compound tubulosaccular glands in the submucosa; a reinforcement of parallel bundles the compact tissues between the serous and the external longitudinal muscle. A small intestine with abundant villis and without paneth cells. The cecum in his luminal face, present a spiral pleat. The histological comparative analysis, allowed to establishing that the basic structure of the wall of the tubular digestive tract is similar to described in other species.

KEY WORDS: Ostrich; Histology; Digestive system.

\section{REFERENCIAS BIBLIOGRÁFICAS}

Angel, C. R. A review of ratite nutrition. Animal Feed Science and technology, 60:241-6, 1996.

Asociación Gremial de Criadores de Avestruces de Chile. Anatomía del avestruz. http://www.acac.cl/fia/fiamain.htm, abril, 2005.

Bacha, W. J. \& Wood, L. M. Aparato digestivo. En: Atlas color de Histología Veterinaria. Buenos Aires, Inter-Médica, 1991.

Calhoun, M. L. Microscopic anatomy of the digestive system of the chicken. Iowa State University Press, Ames, 1954.

Camiruaga, M. \& Simonetti, C. Avestruces, Sistema Digestivo y su Alimentación. Rev. Agronomía y Forestal UC, 12:10-4, 2003.

Camiruaga, M. El avestruz. Sistema de producción en Chile. Fundación para la innovación agraria. Santiago, FIA, 9-25, 2004.

Catroxo, M. H. B.; Lima, M. A. \& Cappellaro, C. E. Histological aspects of the stomach (Proventriculus and gizzard) of the redcapped cardinal (Paroaria gularis gularis, Linnaeus, 1766). Rev. Chil. Anat., 15 (1):19-27, 1997.

Cooper, R. \& Mahroze, K. Anatomy and physiology of the gastrointestinal tract and growth curves of the ostrich (Struthio camelus). Animal Science J., 75:491-8, 2004.

Crossley, J. Fisiología: Particularidades funcionales del Avestruz. Resumen de presentaciones. Curso Especializado en Producción de avestruces: nutrición, manejo y patología. Universidad Santo Tomás, Santiago, Chile, 2001.

De Oliveira, S. \& Inforzato, M. Histological aspects of the stomach of burrowing owl (Speotyto cunicularia, MOLINA, 1782). Rev. Chil. Anat., 16(2):191-7, 1998.

Deeming, D. C. Introducción. En: El Avestruz: Biología, producción y sanidad. Zaragoza, Acribia, 2001. pp1-11.

Delhon, G. A; Fernández-Surribas, J.; Gagna, G.; Lacolla, D. \& von Lawzewitsch, I. Aparato digestivo de las aves domésticas. En: Lecciones de histología veterinaria. Hemisferio Sur, Buenos Aires, 1984. pp. 45-111.
Dieter Dellmann, H. \& Eurell, J. Textbook of Veterinary histology. $5^{\text {th }}$ ed. Philadelphia, Lippincott Williams \& Wilkins, 1998.

Habel, R. E. Sistema digestivo de los rumiantes. En: Sisson, S.; Grossman, J. D. Anatomía de los animales domésticos. Madrid, Masson, 2000. V. I.

Illanes, J; Vigneaux, I. M.; Adaro, L; Olivares, R; Mendoza, J. \& Riveros, V. Análisis morfológico comparado de la región fúndica entre un cánido (Canis familiaris) y un camélido suramericano (Lama glama). Rev. Chil. Anat., 12(2):161-7, 1994.

Luna, L. Manual of histologic Staining methods of the Armed forces Institute of Pathology. 3. ed. Mc Graw-Hill, New York, 1968.

McLelland, J. Sistema digestivo de las aves. En: Sisson, S.; Grossman J. D. Anatomía de los animales domésticos. Madrid, Masson, 2000. V. II. pp. 2035-2063

Sisson, S. Sistema digestivo de los equinos. En: Sisson, S. y Grossman J. D. Anatomía de los animales domésticos. Madrid, Masson, 2000. V. 1. pp. 507-56.

Sociedad Comercializadora De Avestruces S.A. Seminario: Aspectos Económicos y Fisiológicos del Avestruz. Santiago, Comertruz, 2003.

Stinson, A. W. \& Calhoun, M. L. Sistema Digestivo. En: Dieter Dellmann, H. Histología Veterinaria. Zaragoza, Acribia, 1993. pp. 239-56.

Swart, D.; Rahn, H. \& de Kock, J. Nest microclimate and incubation water loss of eggs of the African ostrich (Struthio camelus var. domesticus). J. Exp. Zool. Supplement., 239-46. 1987.

Direccion para Correspondencia:

Prof. Dr. Iulio Illanes Herrero

Departamento de Morfologia

Facultad de Medicina, Clinica Alemana-Universidad del Desarrollo

Av. Las Condes 12.438, Lo Barnechea.

Santiago-CHILE

e-mail:jillanes@udd.cl

Recibido : 03-02-2006

Aceptado: 10-03-2006 